\title{
„Missbrauch“ in der Frühförderung
}

\author{
Heide Simoni
}

Die Prävention und ggf. Erkennung von Missbrauch ist gegenüber allen Kindern eine wichtige Verantwortung von Erwachsenen. Zum Thema Kindesschutz in der frühen Kindheit gibt es verschiedene empfehlenswerte Publikationen, wie bspw. die Broschüren der Stiftung Kinderschutz Schweiz oder der GAIMH. Kinder mit einem Frühförderbedarf können aufgrund charakteristischer Besonderheiten als eine Gruppe gelten, die besonders verletzlich gegenüber der Missachtung ihrer Integrität sowie ihrer Rechte auf Schutz und Selbstbestimmung ist.

In der Frühförderung sind Kinder, die aufgrund individueller Beeinträchtigungen und damit einhergehender Entwicklungsdefizite oder -verzögerungen erstens besonderen Unterstützungsbedarf haben, was sie abhängiger vom Umfeld macht als andere Kinder. Oft sind viele verschiedene Personen involviert und die Zeitspanne intensiver Betreuung ist erhöht und verlängert. Die besonderen Voraussetzungen der Kinder prägen zweitens oft ihre Beziehungen und ihr Beziehungsverhalten und damit ihr Verhältnis zu ihren Bezugspersonen in der Familie und deren Umfeld, zu involvierten Fachpersonen sowie zu anderen Kindern und manchmal auch zu ihnen völlig fremden Personen. Eventuell ist drittens durch die Beeinträchtigung oder das Erleben und Verhalten der Erwerb von Kompetenzen, die für den Selbstschutz wichtig wären, erschwert oder unmöglich. Dazu gehören die selbstständige Körperpflege, Ernährung und Fortbewegung, die Regulierung von Nähe und Distanz, die Fähigkeit, sich kognitiv in andere hineinzuversetzen und Absichten zu erkennen, sowie ganz wichtig: der Erwerb der verbalen Sprache und eines episodischen Gedächtnisses. Beides ist jedoch für Abgrenzung und Selbstbehauptung sowie für die Erinnerung von Ereignissen und für Beschwerden unerlässlich.
Der besondere Unterstützungsbedarf eines Kindes wird manchmal durch psycho-soziale Belastungen ihrer Familien verstärkt bzw. sogar mitverursacht. Es kann also viertens eine Gefährdung des Kindes durch eine anhaltende Überforderung der Bezugspersonen bestehen. Die Besonderheiten und der Bedarf des Kindes können fünftens zudem selbst kompetente und belastbare Bezugs- und Betreuungspersonen emotional und praktisch zeitweise überfordern. Die Überforderung der für ein Kind verantwortlichen Erwachsenen erhöht das Risiko von Kindsmisshandlungen jeder Form erheblich.

Eine verbandsübergreifende Schweizer Arbeitsgruppe hat 2011 eine Charta zur Prävention sexueller Ausbeutung, Missbrauch und anderen Grenzverletzungen erarbeitet, die mittlerweile von vielen Verbänden, Institutionen und Organisationen, die im Behindertenbereich tätig sind, unterzeichnet wurde. Die Charta umfasst 10 Grundsätze, welche die vier Themenbereiche Präventionskonzepte, Stärkung der Personen mit Unterstützungsbedarf, Schlüsselrolle der Mitarbeitenden sowie die Einrichtung einer internen Meldestelle und externen Ombudsstelle betreffen. Die formulierten Grundsätze gelten für alle Personen, die in Institutionen oder Organisationen tätig sind oder von innen betreut werden. Anlass für die Charta war das Bekanntwerden von Übergriffen, die ein Sozialpädagoge über viele Jahre an behinderten, von inm im institutionellen Kontext (mit-)betreuten Menschen verübt hat. Alle in der Charta erwähnten Punkte lassen sich jedoch unschwer auf junge Kinder und ambulante Settings übertragen. So ist es auch für Fachdienste der Frühförderung wichtig, die Prävention von Misshandlung und die Früherkennung entsprechender Vorfälle zu reflektieren, konzeptuell zu verankern, entsprechend umzu- 
setzen und zu überprüfen. Ein großer Stellenwert kommt dabei der Aufmerksamkeit gegenüber kritischen Situationen und der Reflexion des eigenen Verhaltens auf der Leitungs- und Teamebene zu. Für den konkreten Verdachtsfall ist es nützlich, zwischen Risikomanagement und Krisenmanagement, die je mit anderen Handlungsmöglichkeiten und -pflichten verbunden sind, zu unterscheiden (vgl. Text der Fachstelle Limita). Ein Risikomanagement geht mit Qualitätssicherung und Prävention einher und kommt bei Beschwerden und Übertretungen im Graubereich zum Tragen. Das Krisenmanagement ist bei Beschwerden mit Verdacht auf sexuelle Delikte unerlässlich und geht immer mit einer Intervention einher. Nötig sind dabei eine sorgfältige Planung des Vorgehens und ggf. eine Anzeige. Die Konfrontation des möglichen Täters/ der möglichen Täterin sowie Alleingänge sind unbedingt zu unterlassen. Risikomanagement und Krisenmanagement sind Chefsache.

Die Stärkung des Selbstschutzes und die Unterstützung des Aufbaus entsprechender Kompetenzen können und müssen bei jedem Kind in der Frühförderung von fachlicher Seite gezielt unterstützt werden. Manchmal sind es gedankenlose Bevormundungen der familialen und professionellen Bezugspersonen, die deren Entwicklung (zusätzlich) bremsen. Erfahrungen eigener Selbstwirksamkeit sind jedoch eine unabdingbare Basis, um sich selbst schützen oder zumindest dazu einen Beitrag leisten zu können.

Schließlich gilt es der Abhängigkeit und Verletzlichkeit von Kindern durch Triangulierung zu begegnen. Kinder mit einem erhöhten oder besonderen Unterstützungsbedarf sollten hellhörige Vertrauenspersonen im nahen und weiteren Umfeld kennen. Sie sollten so gut wie möglich über ihre Rechte informiert sein und wissen, an wen sie sich wenden können (wer sie hört!), wenn sie bedrängt werden oder sich ängstigen. Selbst junge Kinder und Kinder mit Beeinträchtigungen sind empfänglich dafür und können durchaus begreifen, worum es im Wesentlichen geht. Bspw. kann mit einem Ampelsystem zu Behandlungs- oder
Betreuungsstandards auf anschauliche Weise vermittelt werden, was für Betreuungspersonen konkret verboten (rot), was kritisch (orange) und was erlaubt/erwünscht ist (grün).

Eine entwicklungsentsprechende, respektvolle Kommunikation bildet die Basis für den Erwerb von Selbstvertrauen und für eine gesunde Selbstbehauptung. Es ist erstaunlich, wie oft die direkte Ansprache von jungen Kindern - gerade dann, wenn sich verschiedene Bezugs- und Betreuungspersonen begegnen - vergessen geht. Dabei wäre es gar nicht so schwierig, "mit dem Kind statt über das Kind" zu reden.

\section{Dr. Heidi Simoni}

Marie Meierhofer Insitut für das Kind

Pfingstweidstr. 16

$\mathrm{CH}-8005$ Zürich

simoni@mmi.ch

\section{Literatur}

Bundesministerium für Familie, Senioren, Frauen und Jugend, Deutschland (2013): Qualitätsstandards für die Beteiligung von Kindern und Jugendlichen

Brunner, S. (2013): Früherkennung von Gewalt an kleinen Kindern. Leitfaden für Fachpersonen im Frühbereich. Bern: Stiftung Kinderschutz Schweiz

Charta zur Prävention sexueller Ausbeutung, Missbrauch und anderen Grenzverletzungen (2011): http://www.charta-praevention.ch/userfiles/ downloads/Charta_Praevention_D_A4.pdf (Zugang: 4.1.2016)

Fachstelle Limita (2015): Beschwerden erleichtern Risiken senden - Krisen bewältigen. Leitartikel im Jahresbericht

Thoms, E., Salgo, L., Lack, K. (2014): Kindesschutz in der frühen Kindheit. Ein Leitfaden für die Praxis. Psychosozial Verlag. (Adaptierte Fassung der Kindesschutzbroschüre der GAIMH für D)

Specht, H. (2015): Verdacht auf Missbrauch von Macht. Fürsorgepflichten des klärenden Systems, in DGFPI Fachzeitschrift Kindesmisshandlung und -vernachlässigung 2/2015 